\title{
Interface Effects on Tree Growth of Cross-linked Polyethylene
}

$\begin{array}{lll}\begin{array}{l}\text { Member, } \\ \text { Student-member, }\end{array} & \text { Noboru Yoshimura, Changmin, } & \text { (Akita University) } \\ \text { Member, } & \text { Emi Hirade, } & \text { (Akita University) } \\ \text { Member, } & \text { Hiroyuki Miyata, } & \text { (Akita University) } \\ \text { Member, } & \text { Toshio Niwa, } & \text { (Fujikura Ltd) } \\ \end{array}$

\begin{abstract}
It is well known that treeing is one of the main causes of breakdown of CV cables. It has been also considered that the interface exists between two solid insulation materials become a weak point on the electrical strength of $\mathrm{CV}$ cables. Therefore, it is important to reseach on the tree growing properties when there exists an interface. For evaluating the interface effect on the $\mathrm{AC}$ tree growth, an experimental investigation has been carried out by preparing an interface between two crass-linked polyethylene(XIPE) blocks. This paper describes the experimental results under various interface conditions. It was found that the interface had a great influence on the tree growth The time to breakdown was reduced by the interface in the present test conditions. Painting the silicone oil on the interface, not only reduced the time to breakdown but also prevented the breakdown through the surface of specimen(B). And the pressure applied on the interface had a slight effect on the time to breakdown but on the tree growth pattern. With the increasing of applied voltage, phenomena above mentioned were more significant. It is proposed that the discharge column activities and the gas pressure reduction in the tree channels play important roles in the present situation.
\end{abstract}

Key words: Electrical tree, Interface, Interface pressure, Gas discharge, Discharge column

\section{Introduction}

In spite of the fact that cross-linked polyethylene (XIPE) insulated cable( $\mathrm{CV}$ cable) has established an excellent service record, it had been once reported that some of $\mathrm{CV}$ cables failed in service over several years. This might be because the insulation of the $\mathrm{CV}$ cables was deteriorated by trees initiated and grown from voids in XIPE and interfaces between XIPE and other insulating materials under a high electric field In fact, careful microscopic analysis of the $\mathrm{CV}$ cables broken down in sevice has found various types of trees.

Recently, it has been known that the interface prepared between two materials plays an important role in electrical insulation resistance. Therefore, it is important to investigate the electrical strength of the interface because it seems that the interface prepared between two materials becomes a weak point on the electrical strength of $\mathrm{CV}$ cables.

A trial was performed on evaluating the electrical strength of an interface prepared between two XIPEs. In this paper, we describe the experimental results for the interface's effect on electrical tree-growth in XIPE when an ac voltage with $50 \mathrm{~Hz}$ was applied. It was found that the interface prepared between two XIPEs had a great influence on tree growth.

\section{Experiments}

A XILPE block $(12 \times 13 \times 4 \mathrm{~mm})$ was used as a specimen After the specimen was heated for 15 minutes at $140^{\circ} \mathrm{C}$, a needle (ground by Ogura Jewel Industry $C_{0}$.) with tip angle of $30^{\circ}$ and curvature radius of $5 \mu \mathrm{m}$ was then inserted into it. The distance between the needle tip and the plane electrode(painted conductive silver) was $2 \mathrm{~mm}$ without the interface, as shown in Fig.1(a). On the other hand, for the specimen with the interface the distance between the needle tip and the bottom of the specimen $A$ was $1 \mathrm{~mm}$ and the thichness of specimen B was $1 \mathrm{~mm}$ as shown in Fig.1(b).

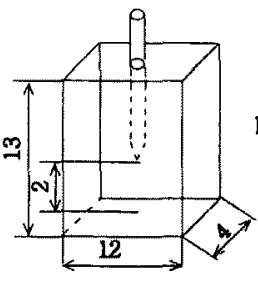

(a) Iithout interface Fig.1 Configuration of specinen(m)
The specimen set on the apparatus shown in Fig.2 was 
immersed in silicone oil to prevent the surface flashover. The $50 \mathrm{~Hz}$, ac voltage was increased at a constant rate of about

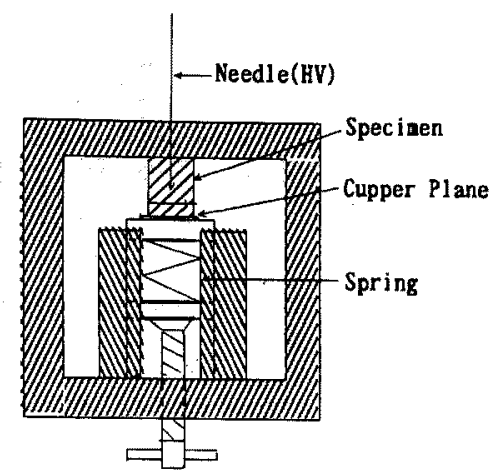

Fig.2 Experiment apparatus

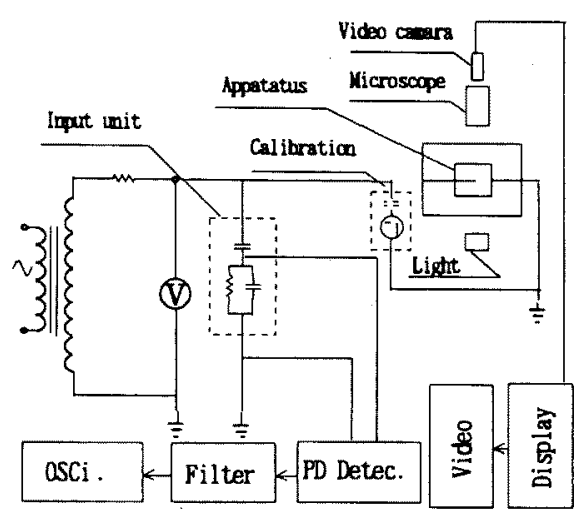

Fig.3 Diagram of experimental set-up

$1 \mathrm{kV} / \mathrm{sec}$ to a certain voltage, and then the growth and the shape of tree were observed on a display through an optical microscope ( $\times 40)$, a CCD camera and a video tape recorder. The magnitude and phase-PC of PD was measured simultaneously. The experimental set-up diagram is shown in Fig. 3.

At present study, the following conditions were chosen:

(1) Three kinds of applied voltage, those were 10, 11 and $12 \mathrm{kV}$. The applied time of voltage was till 120 minutes.

(2) Four kinds of interface pressure(the pressure applied on the interface), it was varied in the range from $1 \mathrm{~kg} / \mathrm{cm}^{2}$ to $20 \mathrm{~kg} / \mathrm{cm}^{2}$. The interface pressure was controlled by a spring length of experimental apparatus shown in Fig.2. The interface pressure was measured by pre-scale(Fuj-Film Co.).

(3) With or without the silioone oil at the interface. Silicone oil was painted on the interface just before the test starting.

It has been reported that the difference of time to breakdown among varoius surface's roughnesses wasn't observed[2]. Therefore, in this study we kept the surface's roughness ground by sandpaper of \#1000 as constant

\section{Experimental Results}

\subsection{The general aspect of tree-growth pattern}

A typical aspect of tree growth and its shape when the interface is prepared between two XIPEs are shown in Fig.4. When a voltage is applied, tree initiates and grows toward the interface. About 10 minutes later, tree reaches the interface and temporarily stops the growth there, and then the tree channels are eroded. Consequently, the tree channels become thick in a few minutes. These phenomena are found for all conditions of the interface prepared. The tree-shape at the first stage when applied $10 \mathrm{kV}$ is shown in Fig.5. After that, however, the growth pattern of tree is divided into three groups depending upon the interface conditions prepared, as

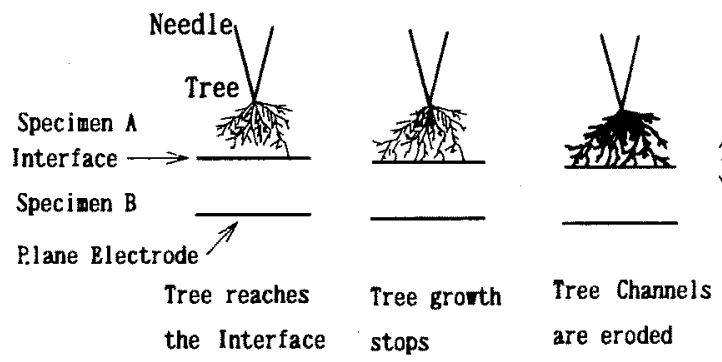

First stage

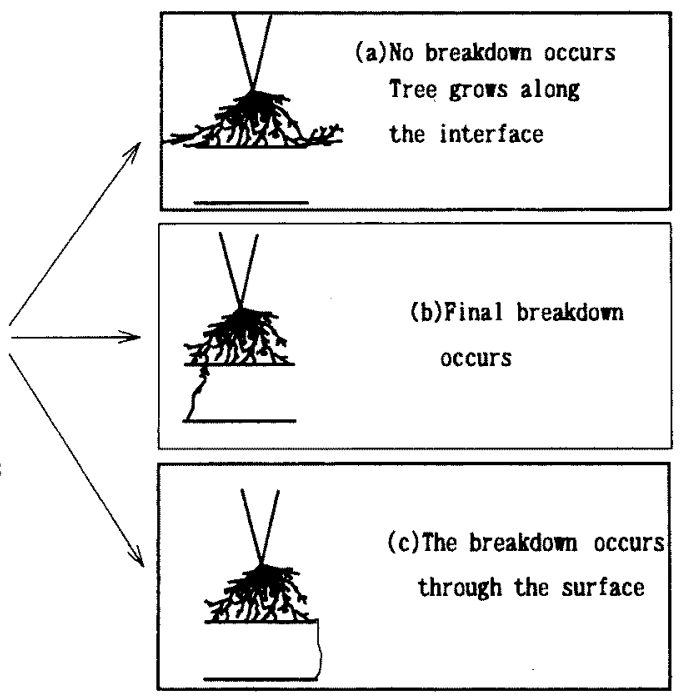

Second Stage

Fig.4 A typical aspect of the growth and the shape of tree 


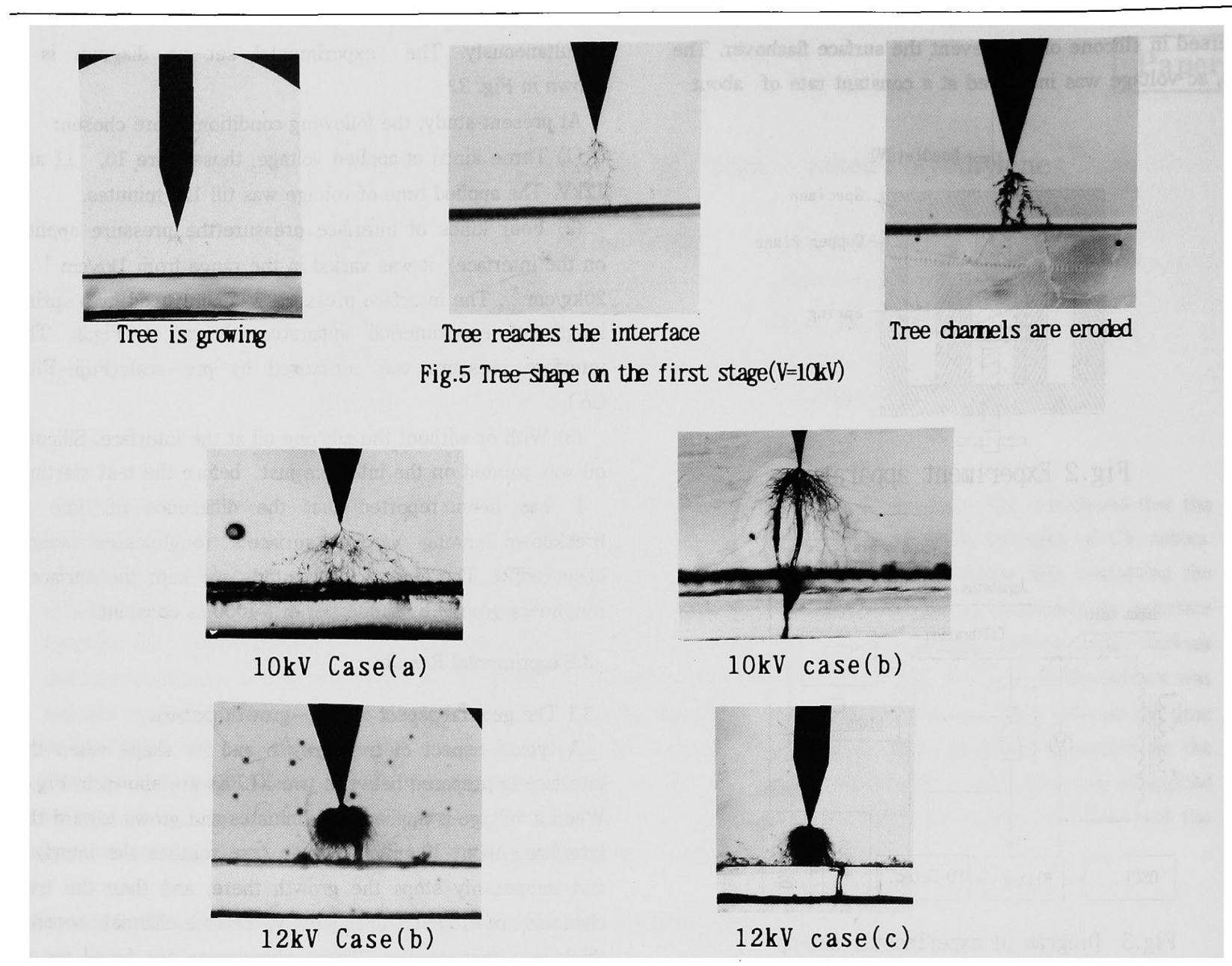

Fig.6 Photographs of second stage

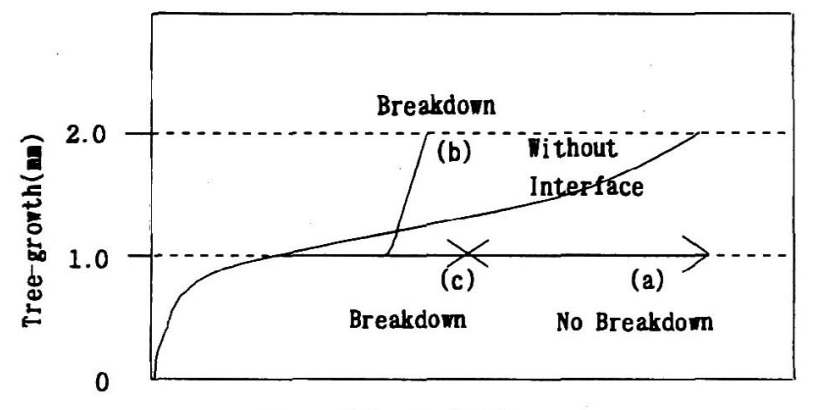

Time of Applied Voltage

Fig.7. A typical tree growth curve

shown in the second stage of Fig.4. First is the case(a) where tree grows along the interface at specimen $A$ side without growing in specimen $B$. In this case, no breakdown occurs even in the application time of 120 minutes. The second is the case(b) where the tree grows into specimen $B$ and puncture breakdown occurs. On the other hand, (c) is the third case. In the case(c), tree grows along the interface, and finally breakdown occurs through the surface of specimen $B$. Fig.6 shows the photographs of the case(a), (b) and (c) under various applied voltages, respectively. A typical tree-growth curve is shown in Fig.7.

Table 1 Probabilities of tree pettem at second stage (a) untr veroivs apied valtzes (\%)

\begin{tabular}{|c|c|c|c|}
\hline $\begin{array}{c}\text { pattern } \\
\text { voltage }\end{array}$ & (a) & (b) & (c) \\
\hline $10 \mathrm{KV}$ & 5 & 95 & 0 \\
\hline $11 \mathrm{kV}$ & 4 & 67 & 29 \\
\hline $12 \mathrm{VV}$ & 0 & 39 & 61 \\
\hline
\end{tabular}

(b) under varoils interface presures $(\%)$

\begin{tabular}{|c|c|c|c|c|c|c|c|c|c|}
\hline & \multicolumn{3}{|c|}{ (a) } & \multicolumn{5}{c|}{ (b) } & \multicolumn{3}{c|}{ (c) } \\
\hline $10 \mathrm{KV}$ & 5 & 0 & 0 & 32 & 32 & 32 & 0 & 0 & 0 \\
\hline $11 \mathrm{kV}$ & 4 & 0 & 0 & 24 & 14 & 29 & 14 & 14 & 0 \\
\hline $12 k \mathrm{kV}$ & 0 & 0 & 0 & 4 & 9 & 26 & 26 & 26 & 9 \\
\hline
\end{tabular}

(c) with or withat si-oil paintad ( $($ )

\begin{tabular}{|c|c|c|c|c|c|c|}
\hline oil & \multicolumn{2}{|c|}{ (a) } & \multicolumn{2}{c|}{ (b) } & \multicolumn{2}{c|}{ (c) } \\
\hline voltage & no oil & oil & no oil & oil & no oil & oil \\
\hline $10 K V$ & 5 & 0 & 47 & 47 & 0 & 0 \\
\hline $1 \mathrm{kV}$ & 4 & 0 & 24 & 43 & 29 & 0 \\
\hline $12 \mathrm{kV}$ & 0 & 0 & 17 & 22 & 35 & 26 \\
\hline
\end{tabular}




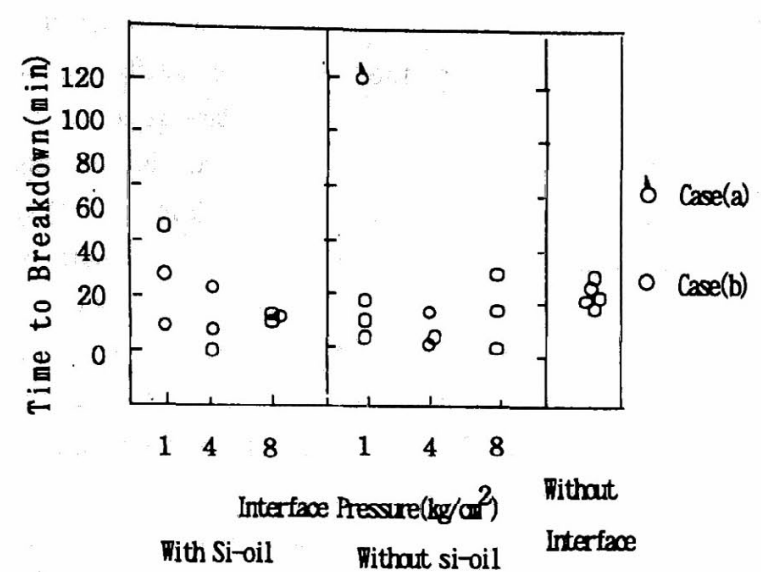

(a) Applied Vol tage: $10 \mathrm{kV}$

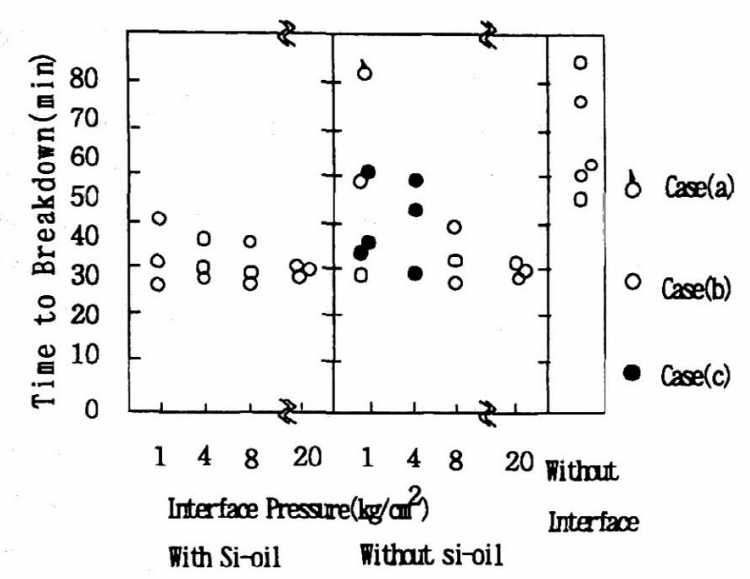

(b) Applied Voltage: $11 \mathrm{kV}$

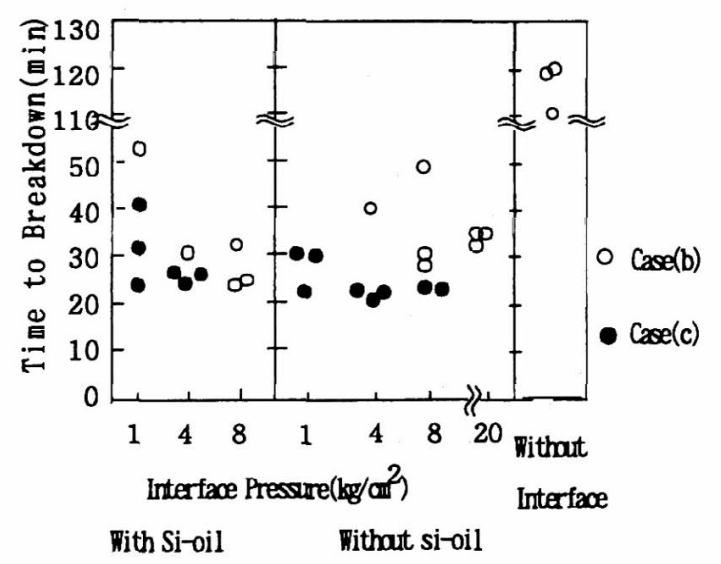

(c) Applied Voltage: $12 \mathrm{kV}$

Fig.8 Breakdown time under various conditions

3.2 Probabilities of case(a), (b) and (c) at the second stage The probabilities of treeing pattem of case(a), (b) and (c) at the second stage under various test conditions are shown in tables 1 . The results can be characterized as follows:

(1) With the increasing of the applied voltage, the probabilities of case(a) and case(b) are reduced, meanwhile the case $(c)$ is increased obviously;
(2) Painting the silicone oil on the interface, the probabilities of case(a) and (c), especially case(c), are reduced; (3) With the increasing of the interface pressure, the probabilities of case(a) and the case(c) are reduced.

\subsection{Characteristics of time to brealkdown}

Figure 8 shows the time to breakdown under varoius test conditions. From the comparison of the results under varoius applied voltages and the interface pressures the following facts are summarized:

(1)The time to breakdown for the sample with the interface becomes shorter than those without the interface;

(2)The difference of the time to breakdown among the interface pressures is not found;

(3)The interface pressure can prevent final breakdown through the surface like $\operatorname{case}(\mathrm{C})$, and the preventation increases with the increasing of the applied voltage;

(4) Painting the silicone oil on the interface, reduces not only the breakdown time, but also the probabilities of case(a) and case(c).

\section{Discussion}

For the specimen with the interface, it is observed that after the tree reaches the interface, many bubbles come out from there as shown in Fig.9. At the same time, the pulse number and the magnitude of PD are increased suddenly as shown in Figs.11 and 12. This phenomenon is observed in all the experiments. As the gas leakes through the interface, the gas pressure in the tree channels is reduced. So the gas discharge is more active. The electric field increased by the discharge column at the tip of old tree branches may be stronger $[3,4,6]$. Consequently, the tree growth may be faster. On the other hand, when the tree arrives at the interface, where exists a thin air or silicone oil layer, the discharge column is diffused as shown in figure 10. This may mean that at the tip of the old tree, the electric field is reduced[1,2]. As a result, the growth of tree is slower.

The growth of tree is controlled by these two factors, one is that at the interface the electric field is increasesd by gas leakege, another is that the electric field is descreased by the diffusion, depending on which one is more significant.

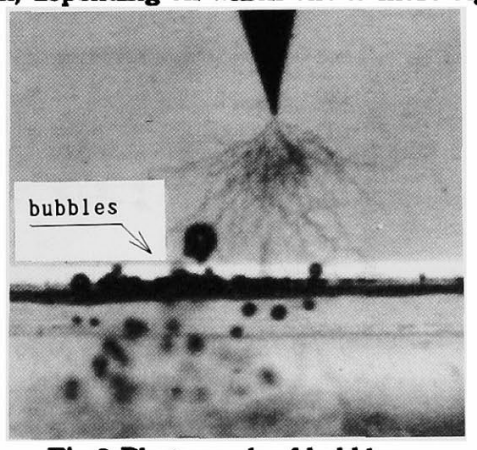

Fig.9 Photograph of bubbles 


\subsection{The effects of the interface pressure}

From the experimental results, the difference of the time to breakdown among the interface pressures varied from $1 \mathrm{~kg} / \mathrm{cm}^{2}$ to $20 \mathrm{~kg} / \mathrm{cm}^{2}$ is not found. But with the increasing of the interface pressure, the tree growth pattern is changed from case(c) to case(b).

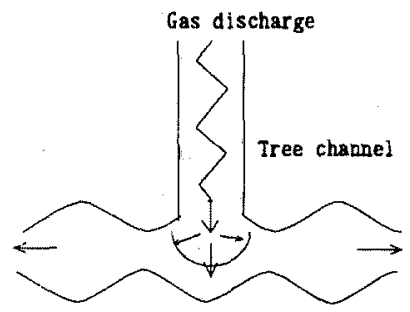

Gas Leakage

Fig.10 Gas discharge model

When the tree reaches the interface, the high voltage is partially transffered by discharge column to there. This divergent field may cause the partially breakdown(tree grow) along the surface. With the increasing of the interface pressure, withstand stress of gas or silicone oil may be increased by the pressure. Therefore, along the interface the growth of tree is slowed. It changes the tree pattern from case(c) to case(b) as shown in Fig.8.

In all of the situations, the bubbles come out from the interface even the interface pressure is $20 \mathrm{~kg} / \mathrm{cm}^{2}$. Therefore, it does not affect the gas discharge. Since the interface pressure does not affect the gas discharge, the time to breakdown is almost the same under present conditions.

\subsection{The effects of the silicone oil painting}

Painting the silicone oil on the interface, because silicone

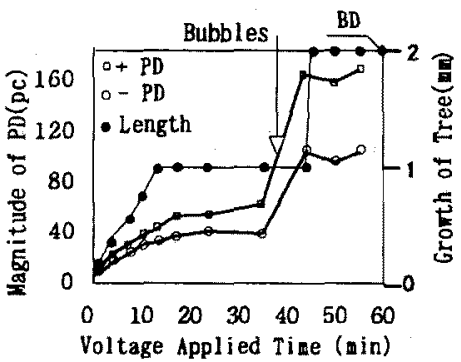

a.TD Vegiturte and Tree Groith vs. Time

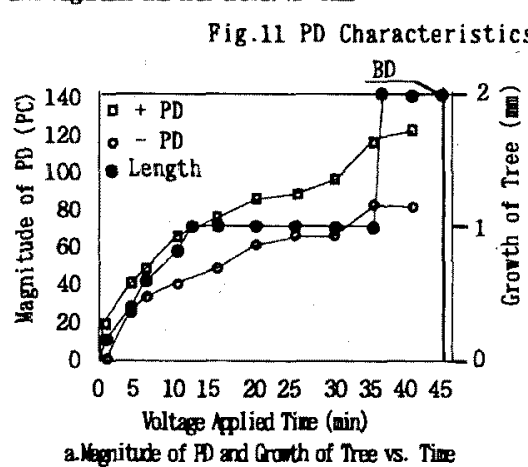

oil has good adhesion for polyethylene, it penetrated into microvoids and defects on the interface's surface. The withstand stress on the surface of the interface is increased and the tree growth along the interface is limited. Consegently, the tree growth pattem changes from case(c) to case(b). On the other hand, because the mean free path or the flowability of the oil must be less than those of air, the diffusion of discharge column may be prevented, and then the maximum electric field at the interface is greater than that without silicone oil. Therefore, the time to breakdown may be shorter than the case of without silicone oil painted.

The typical PD characteristics for the specimens with and without silicone oil painted on the interface are shown in Figs. 11 and 12, respectively. It has been noticed that after large amount of bubbles leaked through the interface, for the specimen without silicone oil painted, the increase of PD magnitude and pulse numbers are more significant. The reason may be that the silicone oil also prevents the gas in the tree channels from being leakage. It is assumed that the increase of the electric field at the the interface by painting the silicone oil on it is more significant, so that the time to breakdown is shorter.

\subsection{The effects of the applied voltage}

When the applied voltage is lower like $10 \mathrm{kV}$, since the gas pressure in the tree channels is relatively lower, the ignition of the gas discharge is easier[3,4]. In this situation, when the tree reaches the interface, the increase of the field increased by gas leakage is not very high. Conseqently, together with the diffusion, the time to breakdown may be a little shorter even be longer as shown in Fig.8.

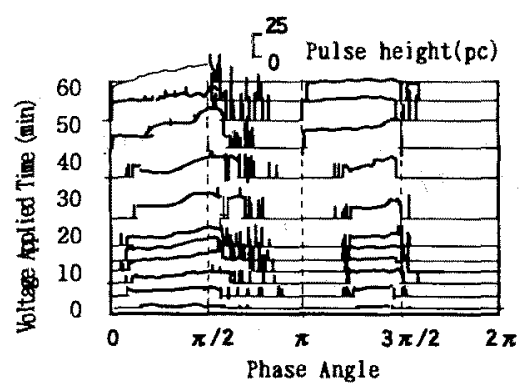

b.Pulse Heigt and Time us. Hese Ngle

$8 \mathrm{~kg} / \mathrm{cm}^{2}$, no oil)

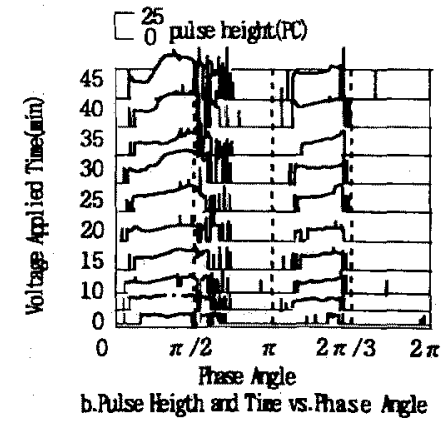

Pig.12 PD Characteristics(10kV, $8 \mathrm{~kg} / \mathrm{cm}^{2}$, oil painted) 
On the other hand, with the increasing of the applied voltage, the gas pressure in the tree channels is higher. When the tree reaches the interface, bubbles come out from there are more, and as a result the discharge column field is higher. By comparison with the diffusion of the discharge column at the interface, the field increased by the gas leakage is more significant. Furthermore, with the increasing of applied voltage, the charge energy of discharge column is higher. The diffusion may be limited. For those reasons, the time to breakdown is extremely shorter than that without the interface as shown in Fig.8.

\section{Conclusion}

(1)The time to breakdown for the samples with the interface becomes shorter compared with those without it

(2)There was not found the change of the breakdown time in spite of the increase of the interface pressure.

(3) The increase of the applied voltage increased the probability of breakdown pattern (case c) through the surface of specimen $B$.

(4)Painting silicone oil on the interface is possible to prevent surface breakdown pattem(case c) and the time to breakdown may be reduced.

\section{Acknowledgement}

The authors wish to thank Dr.M.Suziki and Mr.T.Sato, Akita University for their support of this work.

(Manuscript received Oct. 5,'94, revised Feb. 24, '95)

\section{References}

[1]N.Yoshimura, E,Hirade, H.Miyata and T.Niwa, " Treeing resistance of interface prepared between two cross-linked polyethylenes " 2nd Inter. Conf. Applied Electrostatics, China, Nov.1993

[2]E.Hirade, N.Yoshimura H.Miyata and T.Niwa, "Interface effects on tree-growth in polyethylene under AC stress", 8TH, ISH, 23.08, Yokohama, Aug. 1993

[3]M.Ieda,"Dielectric breakdown process of polymers", IEEE Trans. Vol.EI-15, PP206-224,1998

[4]R.Patsch,"Brakdown of polymer: tree initiation and growth", CEIDP, PP323-334,1975

[5]C.Laurent and C.Mayoux,"Analysis of the propagation of electrical treeing using optical and electrical methods", IEEE Trans.Vol.EI-15, No.1, PP33-42,1980

[6]F.Noto and N.Yoshimura, "Voltage and frenqency dependence of tree growth in polyethylene", Annual, CEIDP, PP207-217,1974

Noboru Yoshimura(Member) He was bom in 1943. He

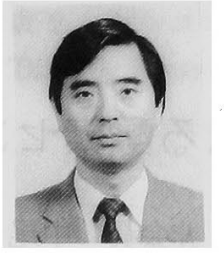
received his B.E. and his M.E. degree in electrical engineering from Akita University in 1967 and 1969 respectively, and his Dr. degree in electrical engineering from Nagoya University in 1975.

Dr. Yoshimura was engaged as an Assistant in the Department of Electrical Egineening, Akita University in 1969, and has been a Professor since 1983, and Dean of Mining College, Akita University since 1995 . He was invited as a Research Associate at Clarkson College of Technology, New York from January, 1978 to September, 1979. His research interests include dielectric materials of polymers and ceramics and semiconductive ceramics.

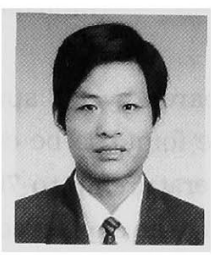

Yuan Changmin(Student-member) He was born in 1960 . He received his B.E. degree in electrical engineering from Xl'AN Jiao Tong University, China in 1984 and M.E. degree in electrical engineering from Akita University in 1995, and still study at the same university as a docotor course student.

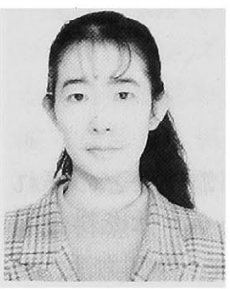

1994.
Emi Hirade(Member) She was bom in 1969. She received a B.E. and M.E. degree in electrical engineering from Akita University in 1992 and 1994 respectively.

She joined Akita Polytechnic College in

Hiroyuki Miyata(Member) He was born in 1957. He

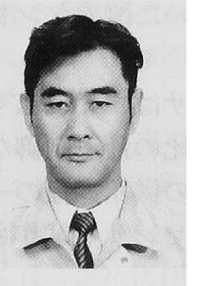
received a B.Sc degree in engineering chemistry from Kobe University in 1981.

He joined Fujikura Ltd. in 1989. He has been engaged in research and development of insulating material of high voltage cables.

Toshio Niwa(Member) He was bom in 1943. He received a

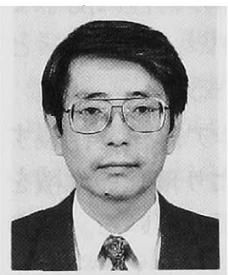
B.Sc. and M.Sc. degree in chemistry from Hiroshima University in 1966 and 1968 respectively, and recieved Dr. in Electrical Engineering from Tokyo Institute Technology in 1994.

He joined Fujikura Ltd. in 1968. He has been engaged in research and development of insulating material of high voltage cables. 